\title{
Universiteit
}

Leiden

The Netherlands

\section{Model calculations of superlubricity of graphite}

Verhoeven, G.S.; Dienwiebel, M.; Frenken, J.W.M.

\section{Citation}

Verhoeven, G. S., Dienwiebel, M., \& Frenken, J. W. M. (2004). Model calculations of

superlubricity of graphite. Physical Review B, 70, 165418. doi:10.1103/PhysRevB.70.165418

Version: $\quad$ Not Applicable (or Unknown)

License: $\quad$ Leiden University Non-exclusive license

Downloaded from: https://hdl.handle.net/1887/61377

Note: To cite this publication please use the final published version (if applicable). 


\title{
Model calculations of superlubricity of graphite
}

\author{
Gertjan S. Verhoeven, Martin Dienwiebel,* and Joost W. M. Frenken \\ Kamerlingh Onnes Laboratory, Leiden University, P. O. Box 9504, 2300 RA Leiden, The Netherlands \\ (Received 26 August 2003; revised manuscript received 12 April 2004; published 26 October 2004)
}

\begin{abstract}
In this paper, friction between a finite, nanometer-sized, rigid graphite flake and a rigid graphite surface is analyzed theoretically in the framework of a modified Tomlinson model. Lateral forces are studied as a function of orientational misfit between flake and surface lattices, pulling direction of the flake, flake size and flake shape. The calculations show that the orientation dependence of the friction provides information on the contact size and shape. We find good agreement between the calculations and the experimental results, discussed in a recent publication by Dienwiebel et al. [M. Dienwiebel, G. S. Verhoeven, N. Pradeep, J. W. M. Frenken, J. A. Heimberg, and H. W. Zandbergen, Phys. Rev. Lett. 92, 126101 (2004)].
\end{abstract}

DOI: 10.1103/PhysRevB.70.165418

PACS number(s): 68.35.Af, 46.55.+d, 07.05.Tp, 07.79.Sp

\section{INTRODUCTION}

Experimental investigations of friction on the atomic scale have become possible by virtue of the friction force microscope $^{1}$ (FFM). In an FFM a sharp tip is scanned with atomic precision over the surface of a sample, while the lateral forces are recorded with a resolution that can be in the $\mathrm{pN}$ range. ${ }^{2}$

Theoretically, atomic-scale friction in the absence of wear, plastic deformation and impurities has been studied using simple ball-and-spring models such as the Tomlinson model, ${ }^{3,4}$ the Frenkel-Kontorova (FK) model,, 5 or a combination of these models, known as the FKT (FrenkelKontorova-Tomlinson) model. ${ }^{7}$ Recently, an extensive overview of the field of computer simulations and theoretical modeling of friction, lubrication, and wear has been given by Robbins and Müser. ${ }^{8}$

In the Tomlinson model, a single atom or a pointlike tip is coupled by a spring to a moving support. This represents the sliding top solid. The bottom solid is treated as a fixed periodic potential energy surface. In a second version of the Tomlinson model, the single atom is replaced by an infinite number of atoms, each connected by a separate spring to the moving, rigid support. In the FK model the atoms in the top surface are coupled to their neighbor atoms by springs, while the coupling to other atoms in the sliding top solid is neglected.

The frictional behavior of such simple ball-and-spring systems has been explored extensively. ${ }^{9,10}$ It has been found that the friction between two crystalline surfaces that slide over each other, in dry contact, but without wear, depends on their commensurability, i.e., whether their lattices share a common periodicity, on the strength of the springs, the strength of the interaction, and on the specifics of the model, such as the dimensionality and the geometry of the springs in the system. Hirano and Shinjo have used numerical calculations for a quasistatic ball-and-spring model of system rigid crystals with fcc, bcc, and hcp symmetry and different orientations, to show that it is possible for incommensurate surfaces, in dry, wearless contact, to slide over each other without phononic energy dissipation, an effect for which they have introduced the name superlubricity. ${ }^{11,12}$

So far, direct comparisons between FFM experiments and model calculations on dry friction have been based mostly on one- or two-dimensional Tomlinson models. Most of these models have involved either pointlike tips ${ }^{13-16}$ or infinite surfaces. ${ }^{17}$ The strengths of the springs ${ }^{15}$ or the normal loads ${ }^{16}$ needed to obtain quantitative agreement with experimental friction force maps of graphite, had to be chosen two orders of magnitude smaller than those in the experiments. To explain this, Morita et al. ${ }^{18}$ have suggested that in FFM experiments on layered materials such as $\mathrm{MoS}_{2}$ or graphite, a flake, consisting of several hundreds of atoms in commensurable contact with the substrate, was attached to the tip.

Recent experimental results by Dienwiebel et al., ${ }^{19}$ obtained by use of a novel FFM, ${ }^{2}$ and discussed more extensively in Refs. 20 and 21, show that the friction force between a tungsten tip and an atomically flat graphite surface was ultralow $(0-50 \mathrm{pN})$ for most relative orientations, except for narrow ranges of orientation where the friction was high (typically $250 \mathrm{pN}$ ). For normal forces in the range between -20 and $40 \mathrm{nN}$, the friction showed only a weak dependence on the loading force.

We interpret this result to be caused by a graphite flake, attached to the tip. ${ }^{19}$ In registry with the substrate, the flake as a whole performs a slip-stick motion during which energy is dissipated, which causes friction. When the flake is rotated out of registry, the forces felt by different atoms start to cancel each other out, causing the friction force to vanish, and the contact to become superlubric.

Lateral forces in finite, nanometer-sized contacts have received little theoretical attention. Total-energy minimization calculations at $T=0 \mathrm{~K}$ of a flat $\mathrm{Cu}(111)$ terminated asperity consisting of 25 to 361 atoms sliding over a $\mathrm{Cu}(111)$ surface have been performed by Sørensen $e t$ al. ${ }^{22}$ Atomic-scale stickslip motion was observed when the two lattices were in perfect registry. For this situation, friction increased linearly with the number of atoms in the contact. When the asperity was rotated $16.1^{\circ}$ out of registry, the friction vanished for sufficiently large contacts. For small contacts, it was found that sliding could result in finite friction, due to a local pinning effect at the corners of the interface.

Sheehan and Lieber have observed that $\mathrm{MoO}_{3}$ nanocrystals in contact with a MoS 2 surface, would slide only along specific directions. ${ }^{23}$ For these directions, a very low shear stress was measured. Using a computer model, they showed 


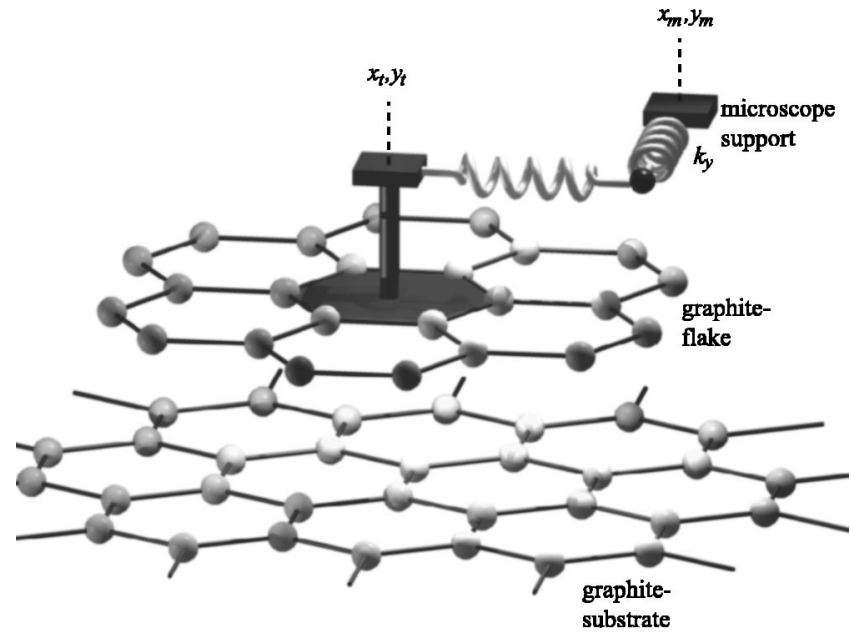

FIG. 1. Illustration of the modified Tomlinson model used in our calculations. A rigid flake consisting of $N$ atoms (here $N=24$ ) is connected by an $x$ spring and a $y$ spring to the support of the microscope. The support is moved in the $x$ direction. The substrate is modelled as an infinite single layer of rigid graphite.

that for a misorientation of $14^{\circ}$ between a rigid $\mathrm{MoO}_{3}$ nanocrystal and $\mathrm{MoS}_{2}$ substrate, the nanocrystal can slide through channels defined by the sulfur atoms of the substrate.

Miura and Kamiya have measured the friction between an $\mathrm{MoS}_{2}$ flake with an area of $1 \mathrm{~mm}^{2}$ and a thickness of several micrometers, and a $\mathrm{MoS}_{2}$ substrate. ${ }^{24}$ In order to interpret the experimental lateral force images, they used a twodimensional Tomlinson model of a pointlike atom that moves through an effective potential that has minima at natural stacking sites of $\mathrm{MoS}_{2}$. They assumed that the flake was always in commensurate contact with the substrate, and obtained qualitative agreement with the experiment.

In the present paper, the friction between a finite, nanometer-sized flake and a graphite surface is analyzed in the framework of a modified two-dimensional Tomlinson model with finite contact size. The graphite flake is modeled as a rigid structure of (pointlike) atoms, and the interaction between the flake and the substrate is assumed to be the sum of all the individual interactions of the atoms.

This paper is organized as follows: Section II describes the model and the methods used. Section III A demonstrates the superlubricity, calculated for a finite-size flake. In Secs. III B and III C, the dependence of the friction and superlubricity on flake size and shape are investigated. Finally, Sec. IV discusses various aspects of the model and compares the results with our experimental observations. ${ }^{19-21}$

\section{MODEL}

The graphite flake is modelled as a rigid, finite lattice, composed of hexagonal carbon rings, as shown in Fig. 1. The flake is coupled to a support by springs in the $x$ and $y$ directions. Via these springs, the support pulls the flake through a periodic potential.

The interaction between a single carbon atom in the flake and the graphite surface is approximated by the interaction potential used in Ref. 14,

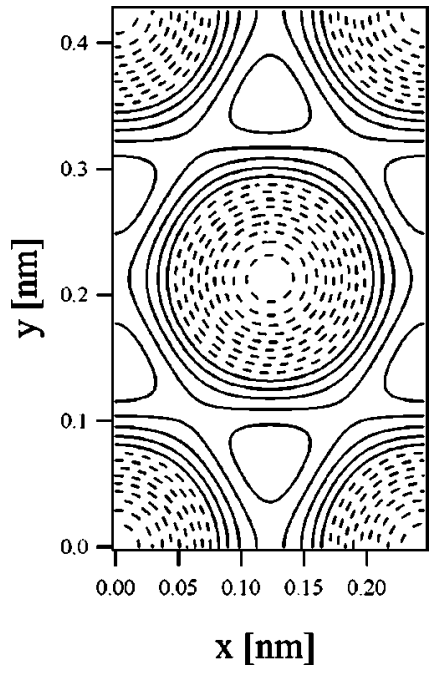

FIG. 2. $0.246 \mathrm{~nm} \times 0.426 \mathrm{~nm}$ rectangular unit cell of the potential energy surface (PES) that describes the interaction between a single carbon atom and the outermost layer of the graphite substrate. The potential has minima of $-3 V_{0}$ and maxima of $1 \frac{1}{2} V_{0}$ (here, $V_{0}=0.032 \mathrm{eV}$ ). Solid and dashed contour lines in the PES denote positive $(V \geqslant 0)$ and negative $(V<0)$ potential energy values, respectively. The contour lines are separated by $0.01 \mathrm{eV}$.

$V_{\text {int }}(x, y, z)=-V_{0}(z)\left[2 \cos \left(a_{1} x\right) \cos \left(a_{2} y\right)+\cos \left(2 a_{2} y\right)\right]+V_{1}(z)$,

with $a_{1}=2 \pi /(0.246 \mathrm{~nm})$ and $a_{2}=2 \pi /(0.426 \mathrm{~nm})$ determined by the periodicity of the graphite surface. The heightdependent corrugation amplitude is given by $V_{0}(z)$, while $V_{1}(z)$ indicates the overall, i.e., position-averaged $z$ dependence of the interaction. Figure 2 shows a contour plot of the potential variations at a constant height $z=c$, i.e., $V_{\text {int }}(x, y, c)-V_{1}(c)$. Equation (1) represents the lowest Fourier components of the interaction between a single atom or a pointlike tip and the first layer of a graphite surface, assuming the potential to originate from pairwise Lennard-Jones interactions. ${ }^{25}$ Expressions for $V_{0}(z)$ and $V_{1}(z)$ can be found in Ref. 24.

Because the relative positions of the atoms in the $N$-atom flake $\left(x_{i}, y_{i}, 0\right)$ with respect to the position $\left(x_{t}, y_{t}, z_{t}\right)$ of the center of mass (CM) of the flake are fixed, the flake-surface interaction potential is simply obtained by the summation over $N$ atomic contributions. The flake can then be treated as a pointlike object moving through this flake-surface potential,

$$
V_{\text {int }}^{\text {flake }}\left(x_{t}, y_{t}, z_{t}\right)=\sum_{i=1}^{N} V_{\text {int }}\left(x_{t}+x_{i}, y_{t}+y_{i}, z_{t}\right) \text {. }
$$

In the experiments, ${ }^{19}$ the FFM was operated at a range of normal loads of up to $F_{N}=+40 \mathrm{nN}$. The system, including the normal force $F_{N}$ can be described by a total potential $V\left(x_{t}, y_{t}, z_{t}\right)=V_{\text {int }}^{\text {flake }}\left(x_{t}, y_{t}, z_{t}\right)-F_{N} z_{t}$. The equilibrium height $z_{t}^{\min }\left(x_{t}, y_{t}\right)$ is given by the minimum of $V\left(x_{t}, y_{t}, z_{t}\right)$ with respect to $z_{t}$. Combining these potential energy values for all positions $\left(x_{t}, y_{t}\right)$, we obtain an effective flake-surface poten- 
tial energy surface $V_{\text {int }}^{\text {flake }}\left(x_{t}, y_{t}\right) .{ }^{10}$ The total potential energy including the elastic energy stored in the springs is given by

$$
V\left(\vec{R}_{t}, \vec{R}_{m}\right)=V_{\mathrm{int}}^{\text {flake }}\left(\vec{R}_{t}\right)+\frac{1}{2} k\left(\vec{R}_{t}-\vec{R}_{m}\right)^{2}
$$

where $\vec{R}_{t}$ is the $\left(x_{t}, y_{t}\right)$ position of the center of mass of the flake, $\vec{R}_{m}$ the $\left(x_{m}, y_{m}\right)$ position of the microscope support, and $k=k_{x}=k_{y}=5.75 \mathrm{~N} / \mathrm{m}$ is the spring constant in the $x$ and in the $y$ direction. Here $k_{x}$ and $k_{y}$ are taken equal to reflect the symmetry of the sensor, employed in the experiments. ${ }^{19}$ The force at the support is given by Hooke's law,

$$
\vec{F}=-k\left(\vec{R}_{t}-\vec{R}_{m}\right) .
$$

In the calculation, all $x$ and $y$ coordinates are discretized in multiples of a basic length unit $l$ of $0.001 \mathrm{~nm}$. This introduces a finite lateral force resolution in the results of $k l=5.75 \mathrm{pN}$ (the experimental error in $F$ was estimated to be $15 \mathrm{pN})$. The calculation procedure is as follows. The support is scanned by displacing it in the pulling direction in steps of $l$. After each step, the position of the flake is allowed to relax towards the nearest local energy minimum. The system is assumed to be in equilibrium at each step of the simulation, i.e., the time scale at which the flake can respond and the time scale at which the excess energy is removed (e.g., carried away by phonons created in the substrate) are assumed to be infinitely short with respect to the time scale of the motion of the support. Although this assumption is not necessarily correct (the time scales could be comparable ${ }^{26}$ ), it provides a useful first approximation to the friction force behavior.

At each position of the support, the energy is minimized by an iterative procedure that moves the flake one length unit $l$ per iteration in the direction of steepest descent in the potential energy. Instabilities in the potential energy surface as a function of flake coordinates $\left(x_{t}, y_{t}\right)$ can cause atomic-scale stick-slip motion, where the flake discontinuously jumps to a new position. Part of the potential energy built up in the springs is removed within a single step of the support, resulting in a nonzero average force, i.e., a friction force, along the pulling direction.

The CM position of the flake initially coincides with the support. Then the support is scanned for the first time over $3 \mathrm{~nm}$ in the pulling direction ( $x$ direction). The system is now considered initialized. The support is then scanned backwards and forwards, again over $3 \mathrm{~nm}$ (the scan size in the experiment ${ }^{19}$ was $3 \mathrm{~nm} \times 3 \mathrm{~nm}$ ). Static friction is defined as the force required in the $x$ direction to cause the first slip event. Kinetic friction is defined as the average force in the $x$ direction after that first event. The area in a closed friction loop equals the total energy dissipated (removed in the energy minimization steps) during the entire loop. Note that the initial slope of each force loop does not equal the stiffness of the spring: within the framework of the model a lateral interface stiffness $k^{\text {interface }}$ exists that is caused by the curvature $\partial^{2} V_{\text {int }}^{\text {flake }} / \partial x^{2}$ at the minima of the periodic potential energy surface. This interface stiffness acts in series with the cantilever springs to produce the effective stiffness that is ob-
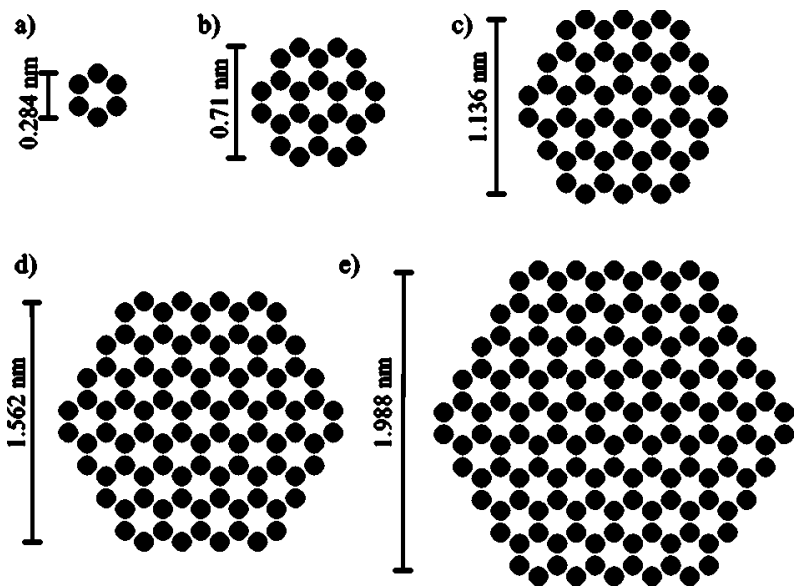

FIG. 3. Symmetric flakes used in the calculations, consisting of (a) 6, (b) 24, (c) 54, (d) 96, and (e) 150 atoms.

served in the simulated friction loops. In the experiments, also the spring coefficient of the tip itself enters the effective stiffness (see Sec. IV and Refs. 20 and 21).

After every combination of one forward plus one backward line in the $x$ direction, the support steps over a distance $6 l(0.006 \mathrm{~nm})$ in the $y$ direction, perpendicular to the pulling direction, and a new forward line is started. In this way, the support also covers a distance of $3 \mathrm{~nm}$ in the $y$ direction, and a two-dimensional lateral force image is generated. Note, that the last flake position in the friction loop is used as the starting position for the next loop, which is more realistic with respect to the experiment than resetting the flake position and initializing it every time, as has been done in several previous studies (e.g., Ref. 14).

The orientation angle $\Phi$ of the flake lattice with respect to the substrate lattice is set prior to calculating the effective interaction potential for the contact. The angle $\Theta$ under which the flake is pulled through the interaction potential is set independently. The friction force for a certain combination of misfit angle $\Phi$ of the contact and pulling direction $\Theta$ of the support is defined here as the average of all kinetic friction values for all different $y$ coordinates within one lateral force map, and also averaging over forward and backward lines.

\section{RESULTS}

\section{A. Superlubricity}

Figure 3 displays symmetric flakes of various sizes that were considered in the calculation. Each flake is a piece of graphene sheet, hereafter loosely referred to as a graphite layer, and has a shape with $60^{\circ}$ rotational symmetry. The friction force (as defined in Sec. II) is maximal if the misfit angle $\Phi$ is zero, i.e., the lattices of flake and substrate form a commensurate structure. For this orientation, the friction force increases linearly with the number of atoms $N$ in the flake. In order to compare different flake sizes for a fixed total interaction between the flake and the surface, the potential amplitude per atom $V_{0}$ was lowered with increasing flake size such that always $V_{0} N=0.52 \mathrm{eV}$. The total interaction 


\section{POTENTIAL ENERGY}
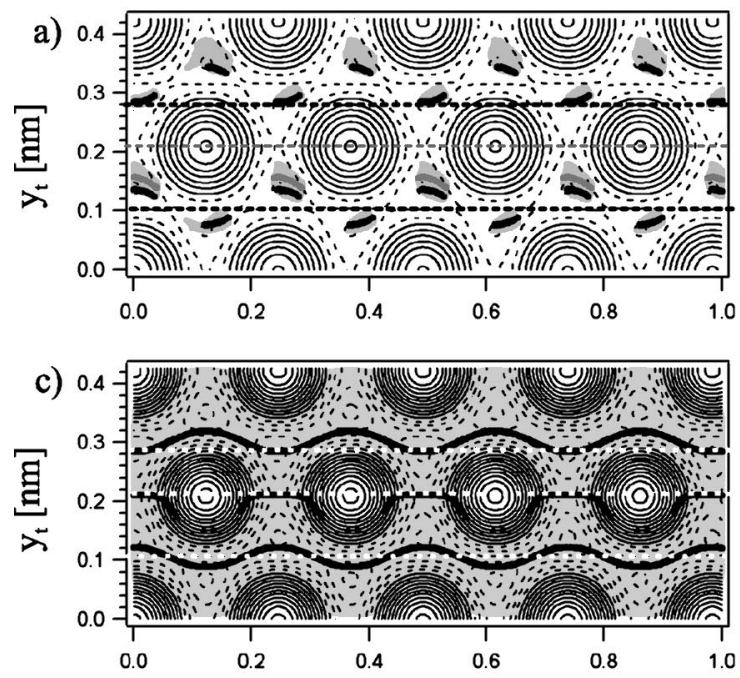

LATERAL FORCE
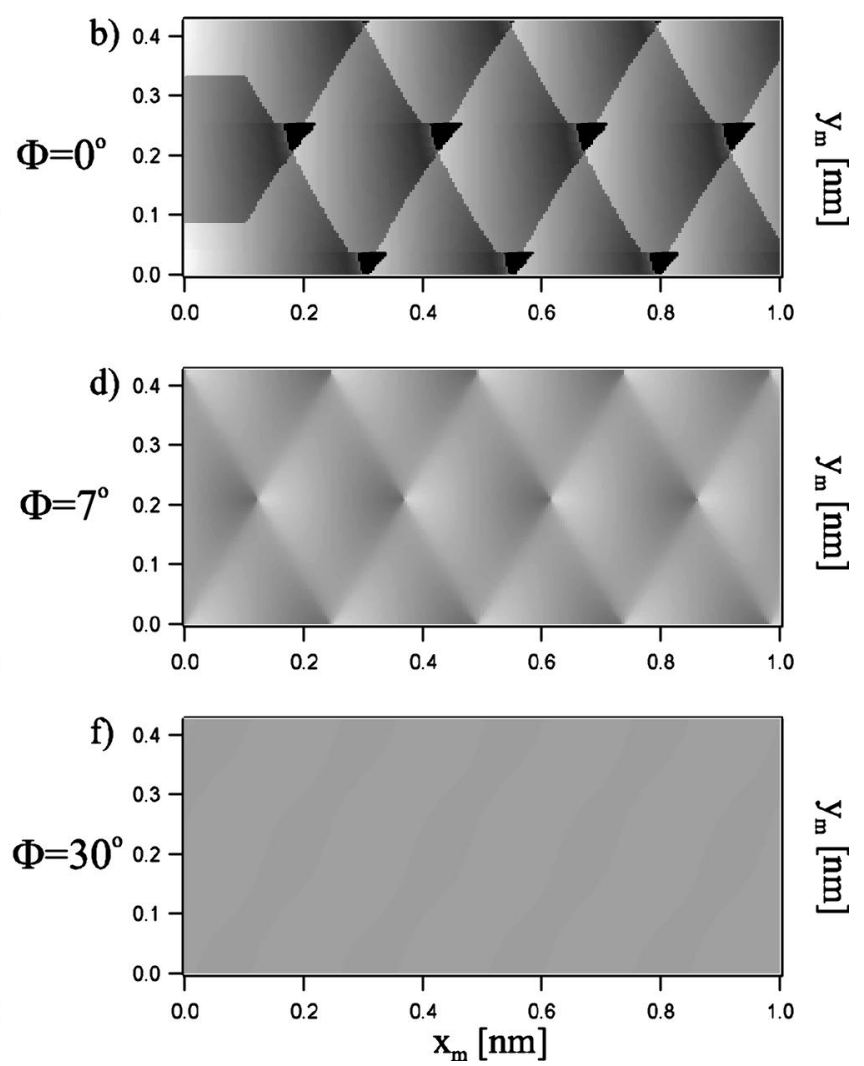

FIG. 4. Total potential energy surfaces and lateral force images $(1.0 \mathrm{~nm} \times 0.426 \mathrm{~nm})$, calculated in the forward $x$ direction for a symmetric, 96-atom flake, for misfit angles $\Phi=0^{\circ}(\mathrm{a}, \mathrm{b}), \Phi=7^{\circ}(\mathrm{c}, \mathrm{d})$, and $\Phi=30^{\circ}$ (e,f). The grey scale in the lateral force images corresponds to the range $[-1.04,0.63] \mathrm{nN}$. For this range, (b) has maximal contrast. Solid and dashed contour lines in the PES denote positive $(V \geqslant 0)$ and negative $(V<0)$ energy values, respectively. The contour lines in (a), (c), and (e) are separated by $0.12 \mathrm{eV}, 0.012 \mathrm{eV}$, and $6.2 \times 10^{-4} \mathrm{eV}$, respectively. The grey areas in the potential energy contour plots denote positions that were visited by the flake. The black lines denote pathways of the flake during single scan lines of the support in the $x$ direction, at $y_{m}=0.104 \mathrm{~nm}, y_{m}=0.212 \mathrm{~nm}$, and $y_{m}=0.284 \mathrm{~nm}$ [indicated with black dashed lines in (a,c), and with white dashed lines in (e)]. In (a), the pathway that belongs to the scan line at $y_{m}=0.212 \mathrm{~nm}$ is colored dark grey to distinguish it from the upper and lower pathways.

energy amplitude $V_{0} N$ was set to $0.52 \mathrm{eV}$, so that the calculated friction force with the flake and substrate in registry was the same for all flakes, namely $265 \mathrm{pN}$ at $0^{\circ}$ pulling direction, corresponding to the value measured experimentally. ${ }^{19}$

The effective interaction potential energy surface (PES) $V_{\text {int }}^{\text {flake }}$ for matching lattices $\left(\Phi=0^{\circ}\right)$ is shown in Fig. 4(a) for $N=96$. Note that in our model, for a commensurate contact, changing the shape of the flake does not affect the calculated friction force. For $\Phi=0^{\circ}$ only the total interaction energy amplitude $V_{0} N$ matters, which has been kept constant here. The positions $\left(x_{t}, y_{t}\right)$ where $V_{\text {int }}\left(x_{t}, y_{t}\right)$ is maximal for a single atom (Fig. 2), are minima of $V_{\text {int }}^{\text {flake }}$ for $\Phi=0^{\circ}$, as displayed in Fig. 4(a) (at $\Phi=0^{\circ}$, our model is similar to that of Miura and Kamiya $^{24}$ ). These minima correspond to flake positions where stacking between the flake and the substrate corresponds to bulk graphite staggering of the graphite planes. This stacking has the effect that only half of the flake atoms have atoms directly below. The other half fall above the centers of the hexagons of the surface below.

The grey areas overlayed on the PES are the flake positions recorded in the $+x$ or forward scan direction, during the
$3 \times 3 \mathrm{~nm}$ scan (of which only $1.0 \mathrm{~nm} \times 0.426 \mathrm{~nm}$ is shown), parallel to the $x$ axis $\left(\Theta=0^{\circ}\right)$. In Fig. 4(a), the flake is only found in limited regions, slightly displaced to the upper right with respect to the minima of the PES. Also shown are flake pathways for three separate scan lines, at $y_{m}=0.104 \mathrm{~nm}, y_{m}$ $=0.212 \mathrm{~nm}$, and $y_{m}=0.284 \mathrm{~nm}$. Friction loops for these pathways are shown in Fig. 5. During the scanning process the flake moves continuously through the grey sticking regions, while force is built up in the spring.

At $y_{m}=0.104 \mathrm{~nm}$, the flake performs zig-zag motion through the PES, with the average force in the $y$ direction $\left\langle F_{y}\right\rangle=0$. Every time that the support is displaced over another lattice spacing, the flake jumps discontinuously to a position $x_{t}>x_{m}$, in front of the support. This results in a positive force $F_{x}$. Only when the support is moved beyond the tip again, does the force switch back to negative. As $y_{m}$ increases, $\left\langle F_{y}\right\rangle$ becomes more negative.

At $y_{m}=0.212 \mathrm{~nm}$, the flake jumps only through the row of PES minima at $y=0.142 \mathrm{~nm}$ and no longer via those at $0.071 \mathrm{~nm}$, resulting in a higher average force in the $x$ direction [Fig. 5(b)]. The average force in the $y$ direction is now negative. It is not before $y_{m} \approx 0.24 \mathrm{~nm}$ that the flake jumps to 

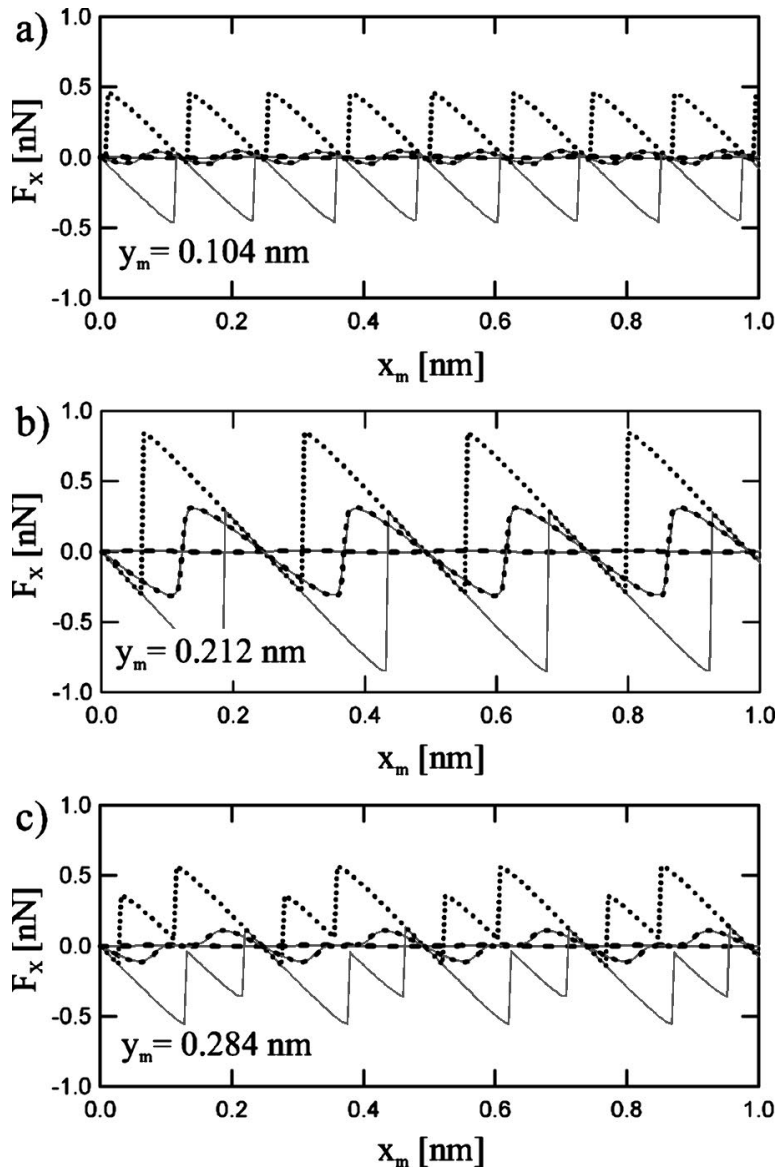

FIG. 5. Calculated friction loops for a symmetric 96-atom flake at rotation angles $\Phi=0^{\circ}, 7^{\circ}$, and $30^{\circ}$ at (a) $y_{m}=0.104 \mathrm{~nm}$, (b) $y_{m}$ $=0.212 \mathrm{~nm}$, and (c) $y_{m}=0.284 \mathrm{~nm}$. The solid lines show the force in the forward $x$ direction, the dotted lines show the force in the backward $x$ direction. In all three panels, the forward and backward forces coincide within the resolution of the plot for $\Phi=30^{\circ}$ (lowestamplitude curves) and $\Phi=7^{\circ}$ (intermediate-amplitude curves). Here, only for $\Phi=0^{\circ}$ the forward and backward curves are visibly separated and energy is dissipated.

the next row of minima at $y=0.284 \mathrm{~nm}$. This may seem surprising at first sight, since $y_{m}=0.213 \mathrm{~nm}$ is located symmetrically on the PES. However, the history of the scan is that previous scan lines were at lower $y_{m}$ values, so that when, for example, $y_{m}=0.220 \mathrm{~nm}$, the flake is still on the lower $y$ side of that symmetry line. This history effect is also reflected in the sharp cuts in the lateral force map [Fig. 4(b)] in the $y_{m}$ region between $0.21-0.24 \mathrm{~nm}$.

Finally, at $y_{m}=0.284 \mathrm{~nm}$, the flake again performs zig-zag motion. Here, the flake motion is not centered around the support scan line, $\left\langle F_{Y}\right\rangle>0$, and two different peak heights appear in the friction force loop [Fig. 5(c)].

When the 96 -atom flake is misaligned by $7^{\circ}$, the calculated friction force (i.e., the average lateral force) vanishes completely $(-0.78 \mathrm{pN})$, within the precision of the calculation $(5.75 \mathrm{pN})$. Figure $4(\mathrm{c})$ displays the calculated effective PES for $\Phi=7^{\circ}$. With respect to $\Phi=0^{\circ}$, the corrugation of the PES has decreased, and the regions addressed by the flake have merged, indicating that the flake moves continuously through most of the PES. Only when the support scans pre-

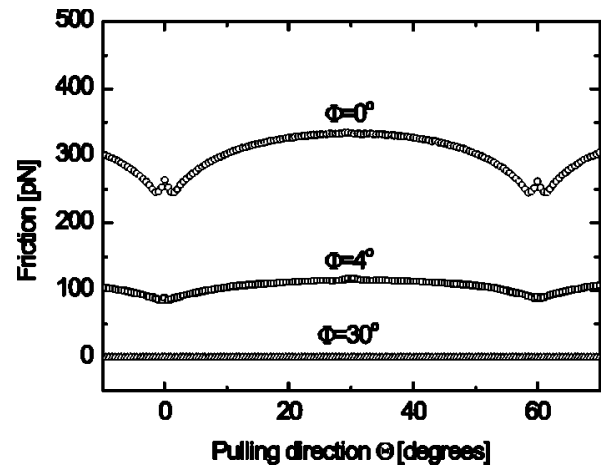

FIG. 6. Calculated friction as a function of pulling direction for three different orientations of a 96-atom flake: $\Phi=0^{\circ}, 4^{\circ}$, and $30^{\circ}$.

cisely over the maxima of the PES, as can be seen in the scan line at $y_{m}=0.284 \mathrm{~nm}$, the flake slips around them. However, for the friction loop recorded at $y_{m}=0.284 \mathrm{~nm}$ [Fig. 5(c)], the small difference of $\left\langle F_{+X}\right\rangle-\left\langle F_{-X}\right\rangle=0.6 \mathrm{pN}$ between the average lateral forces in the forward and reverse pulling direction reveals that even there almost no energy is dissipated.

If the misalignment between the 96-atom flake and the substrate is further increased to $30^{\circ}$, the corrugation of the PES becomes so low that the pathway of the flake through the PES is identical to that of the support, within one length unit $l$ of the calculation. The flake-graphite contact is now completely superlubric.

In order to investigate the dependence on the pulling direction $\Theta$, calculations have been performed for a range of $\Theta$ values for the 96 -atom flake in registry $\left(\Phi=0^{\circ}\right)$, for $\Phi=4^{\circ}$, and for $\Phi=30^{\circ}$. The results are shown in Fig. 6. The maximum variation in the friction force with $\Theta$ was found to be $20 \%-30 \%$ for the commensurate flake as well as for the $4^{\circ}$ incommensurate flake, while the friction was essentially zero for all pulling directions for $\Phi=30^{\circ}$. Choosing a different pulling direction can change the trajectory of the flake. But because the flake still jumps between the same sticking regions via more or less the same saddle points, the friction force depends only modestly on the pulling direction.

A very similar dependence on the pulling direction was found by Gyalog et al. ${ }^{17}$ within a FKT model for two identical infinitely extended crystal surfaces with a square geometry. In contrast with the results in Ref. 18, the friction force in Fig. 6 is lowest at $\pm 1.5^{\circ}$ with respect to the symmetry directions of the graphite surface and slightly higher precisely in the symmetry directions. This is caused by a delicate interplay between the force built up in the $y$ direction in successive scan lines (the history-effect mentioned above), and the force recorded in the $x$ direction. For $\Theta=0^{\circ}$ [Fig. 4(a)], the pathway of the flake during a single scan line is along a single row of PES minima. As $y_{m}$ increases, force is built up in the $y$ direction. This results in higher forces recorded in the $x$ direction, when compared to calculations in which at the start of each new scan line the position of the flake is made equal to that of the support. For angles $0^{\circ}<\Theta<1.5^{\circ}$ between the path of the support and the rows of PES minima, the force in the $y$ direction rises along a single scan line. Still, the scan size of $3 \mathrm{~nm}$ is sufficiently small that the flake jumps to the next row only when the 


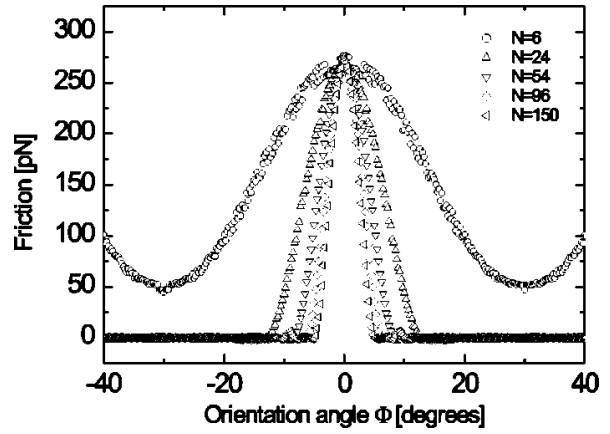

FIG. 7. Friction as a function of the orientation angle for different symmetric flakes ranging in size from 6 to 150 atoms.

support is moved to the next scan line, i.e., when $y_{m}$ is raised. This will occur earlier for increasing pulling angles $\Theta$. Consequently, the average force buildup in the $y$ direction decreases, and the increase of the force in the $x$ direction decreases. Finally, starting at pulling direction $\Theta=1.5^{\circ}$ in Fig. 6 , the flake jumps between rows of PES minima during scan lines, effectively erasing the history of the scan. Note that the pulling direction at which these jumps between neighboring rows start, depends on the length of the scan line. In Fig. 6 the experimental scan size of $3 \mathrm{~nm} \times 3 \mathrm{~nm}$ is used. For increasing scan sizes, the friction minimum moves towards $\Theta=0^{\circ}$, and for infinite scan sizes the dependence of the friction on the pulling direction will equal that of Ref. 18, with the exception of $\Theta$ exactly equal to zero.

\section{B. Flake size dependence}

Figure 7 displays the computed friction force as a function of the misfit angle $\Phi$ (at a pulling direction of $\Theta=0^{\circ}$, for the five symmetric flakes shown in Fig. 3. Because $N V_{0}$ is chosen equal for all flakes, the friction force reaches the same maximum value for $\Phi=0^{\circ}$. We find angular regions with high friction around $0^{\circ}$, repeating every $60^{\circ}$ due to the rotational symmetry of the flakes. At intermediate angles, near-zero friction is calculated, except for the six-atom flake, for which the friction drops to $52 \mathrm{pN}$.

The angular width of the friction maxima should depend on the flake size, because the cancellation of lateral forces can be considered complete when the mismatch between the two lattices adds up to one lattice spacing over the diameter of the flake. This condition provides us with the estimate that

$$
\tan (\Delta \Phi)=1 / D
$$

where $\Delta \Phi$ is the full width at half-maximum (FWHM) of the friction peak, and $D$ is the flake diameter, expressed in lattice spacings. This relation is shown in Fig. 8, where the FWHM of the friction peaks in Fig. 7 is plotted as a function of flake diameter, using the in-plane graphite nearest neighbor distance of $0.142 \mathrm{~nm}$ as lattice spacing. The agreement between the estimate of Eq. (5) and the peak widths calculated for the five flakes is excellent.

\section{Flake shape dependence}

Calculations have also been performed for graphite flakes with shapes that do not have $60^{\circ}$ rotational symmetry. In this

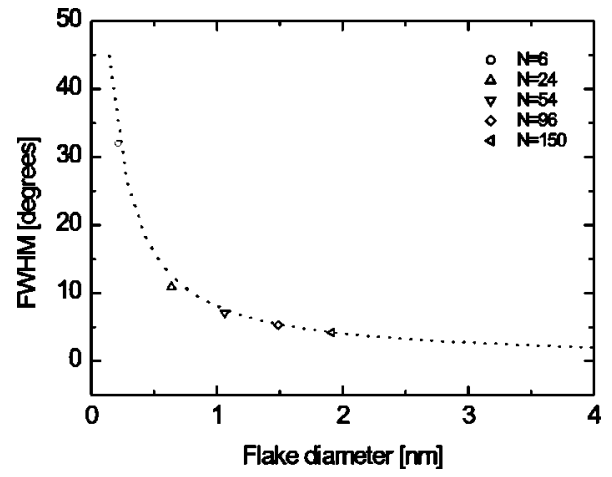

FIG. 8. Width of the friction peaks (FWHM) in Fig. 7 versus flake diameter. The dotted curve is the simple geometrical estimate of Eq. (5).

section, the effect of these so-called asymmetric flakes on the friction is investigated. Taking a 96-atom symmetric flake as a starting geometry, we removed rows of carbon hexagons at the top and at the bottom [Figs. 9(a) and 9(b)] until a single row of carbon hexagons was left [Fig. 9(c)]. This yielded three model flakes with length-over-width ratios of 1.5, 2.4, and 6.1, and consisting of 78,56 , and 30 atoms, respectively. As before, we have kept $N V_{0}$ constant.

Calculated potential energy surfaces and lateral force maps in the forward $x$ direction for the 56-atom flake are shown in Fig. 10. The images were calculated for misfit angles of $\Phi=12^{\circ}(\mathrm{a}, \mathrm{b})$ and $\Phi=30^{\circ}$ (c,d), at a pulling direction of $\Theta=0^{\circ}$. The results for an unrotated flake $\left(\Phi=0^{\circ}\right)$ are again identical to those for the symmetric 96-atom flake, in Figs. 4(a) and 4(b). Due to the stretched shape of the flake, the effective PES becomes elongated along the long axis of the flake when $\Phi \neq 0$. The grey areas show positions that have been visited by the center of the flake during sliding. They reveal that for misfit angle $\Phi=12^{\circ}$, the angle around which the contact becomes fully superlubric, the PES is elongated such, that channels of sticking areas are formed that run across the surface. These low energy channels in which the flake slides continuously, are still separated by energy barriers in the $y$ direction over which the flake must jump. The jump to a new channel causes a sudden shift in the wavy force pattern, vaguely visible in the lateral force image in the $x$ direction. These jumps, however, have a negligible effect on the friction. Low-energy channels, such as calcu- a)

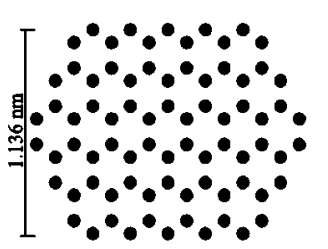

c)

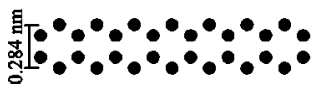

b)

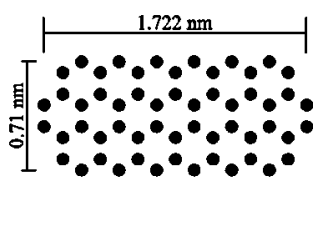

IG. 9. Three asymmetric flakes consisting of (a) 78, (b) 56, and (c) 30 atoms. 


\section{POTENTIAL ENERGY}
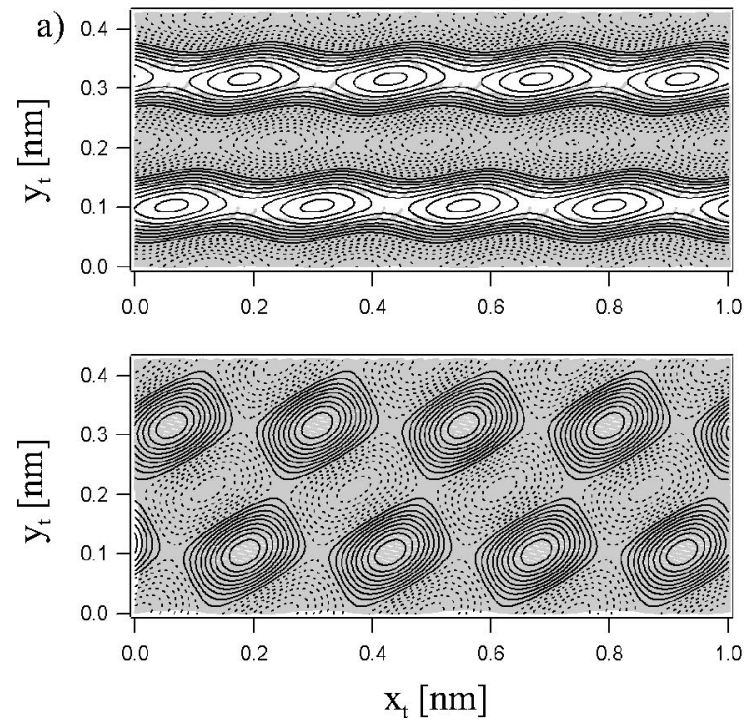

LATERAL FORCE
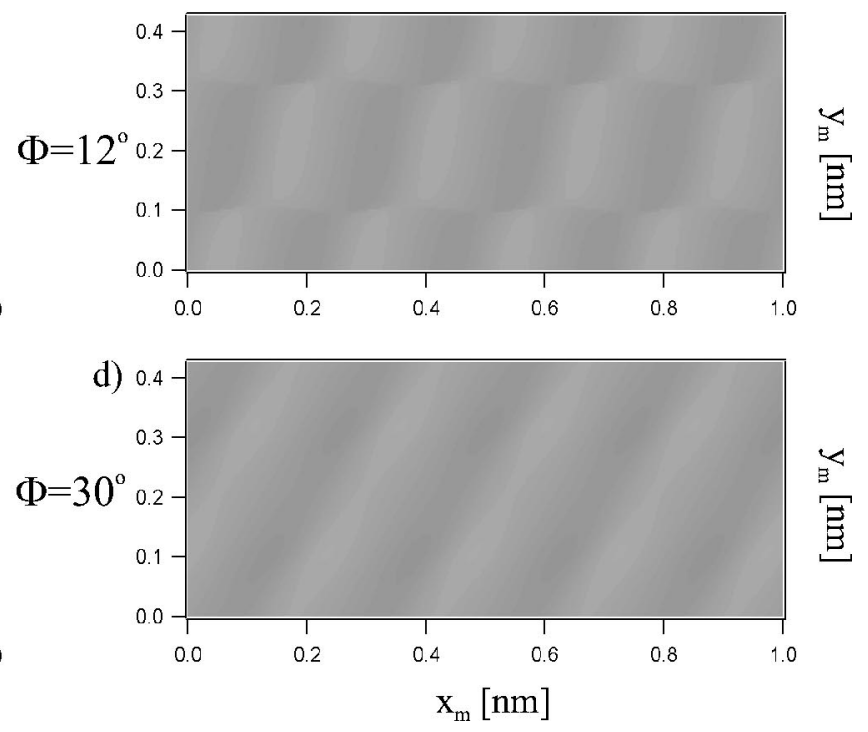

FIG. 10. Total potential energy surfaces and lateral force images $(1.0 \mathrm{~nm} \times 0.426 \mathrm{~nm})$, calculated in the forward $x$ direction for an asymmetric, 56-atom flake, for orientation angles $\Phi=12^{\circ}$ (a,b) and $\Phi=30^{\circ}$ (c,d). Solid and dashed contour lines in the PES denote positive $(V \geqslant 0)$ and negative $(V<0)$ energy values, respectively. The contour lines are separated by (a) $6.2 \times 10^{-3} \mathrm{eV}$ and $(\mathrm{c}) 3.1 \times 10^{-3} \mathrm{eV}$. The grey areas in the potential energy contour plots denote positions that were visited by the flake when scanning in the $x$ direction. The grey scale in the lateral force images corresponds to a force range $[-1.04,0.63] \mathrm{nN}$, equal to that of Fig. 4.

lated here for the rotated asymmetric flake, have been observed experimentally. ${ }^{23}$

Depending on the flake orientation, the sticking zones are elongated in different directions, which creates the impression that the lateral force pattern is rotating [compare Figs. 4(b), 10(b), and 10(d)] although the pulling direction is the same for all lateral force images shown $\left(\Theta=0^{\circ}\right)$. When the flake is rotated $30^{\circ}$ away from commensurability, the PES is at its shallowest and the flake slides continuously over the entire surface.

Figures 11(a)-11(c) shows the dependence of the friction on the misfit angle for $\Theta=0^{\circ}$, for the asymmetric flakes shown in Fig. 9. As for the symmetric flakes, we find regions with high friction that appear every $60^{\circ}$, separated by angular regions that are superlubric. The high friction peaks now exhibit shoulders, which become more prominent the more asymmetric the shape of the flake is. Furthermore, these shoulders are asymmetric, but the pattern shows mirror symmetry with respect to $0^{\circ}$ and $90^{\circ}$. This mirror symmetry is caused by the combination of two elements: (1) for a misaligned asymmetric flake, the shape of the PES causes the flake to follow different pathways in the forward and backward scans. (2) The asymmetric flakes in Fig. 9 possess two mirror planes. This mirror symmetry produces mirrored potential energy surfaces for paired angles

$$
\begin{gathered}
V_{\text {int }}^{\text {flake }}\left(\Phi, x_{t}, y_{t}\right)=V_{\text {int }}^{\text {flake }}\left(-\Phi,-x_{t}, y_{t}\right), \\
V_{\text {int }}^{\text {flake }}\left(90^{\circ}+\Phi, x_{t}, y_{t}\right)=V_{\text {int }}^{\text {flake }}\left(90^{\circ}-\Phi,-x_{t}, y_{t}\right) .
\end{gathered}
$$

For example, Fig. 12 shows the positions in the PES visited by the flake during the forward scan, rotated by $90^{\circ}$ $-26.5^{\circ}=63.5^{\circ}$ (a) and $90^{\circ}+26.5^{\circ}=116.5^{\circ}$ (b), respectively.
The sticking regions in the backward scan for $\Phi=63.5^{\circ}$ (not shown) equal those in Fig. 12(b), but are mirrored in the $y$ axis. Likewise, the sticking regions in the backward scan for $\Phi=116.5^{\circ}$ (not shown), mirrored in the $y$ axis, equal those in

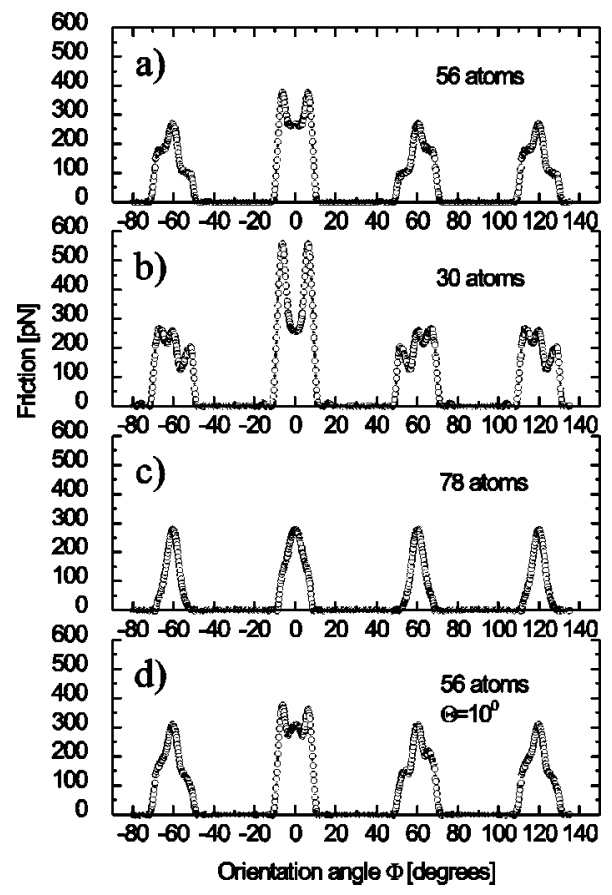

FIG. 11. Friction as a function of the orientation angle for three different asymmetric flakes with $(\mathrm{a}, \mathrm{d}) 56$ atoms, $V_{0}$ $=0.0093 \mathrm{eV}$; (b) 30 atoms, $V_{0}=0.017 \mathrm{eV}$; (c) 78 atoms, $V_{0}$ $=0.0068 \mathrm{eV} .(\mathrm{a}, \mathrm{b}, \mathrm{c})$ are calculations for a pulling direction $\Theta=0^{\circ},(d)$ is calculated at $\Theta=10^{\circ}$. 

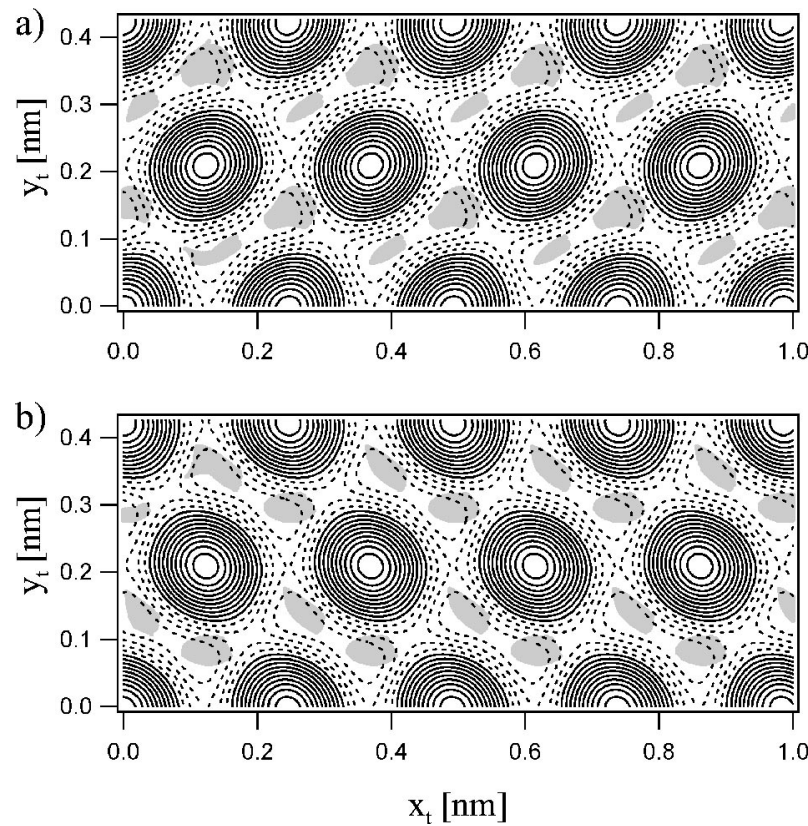

FIG. 12. Calculated effective PES for an asymmetric 56-atom flake for rotation angles $\Phi=63.5^{\circ}$ (a) and $\Phi=116.5^{\circ}$ (b). The grey areas denote positions that were visited by the flake when scanning in the forward $x$ direction. Solid and dashed contour lines in the PES denote positive $(V \geqslant 0)$ and negative $(V<0)$ energy values, respectively. The contour lines are separated by $6.2 \times 10^{-2} \mathrm{eV}$.

Fig. 12(b). Because the friction is defined here as the average of all force values in the backward and forward scans, it follows that for the angle pairs $(+\Phi,-\Phi)$ and $\left(90^{\circ}+\Phi, 90^{\circ}\right.$ $-\Phi)$ equal friction values are calculated for high symmetry pulling directions $\Theta_{M}=0^{\circ}, \pm 60^{\circ}, \pm 120^{\circ}$, etc.

Finally, for pulling directions $\Theta \neq \Theta_{M}$, the friction as function of rotation angle has lost all symmetry, except the $180^{\circ}$ rotation symmetry of the flake, as can be seen in Fig. 11(d) for the 56-atom flake at a pulling direction of $\Theta=10^{\circ}$.

\section{DISCUSSION}

Here, the calculated results are compared with the experimental results. ${ }^{19}$ As a first step in the comparison, we use Eq. (5) or Fig. 8, to obtain an estimate for the flake diameter in the experimental observations. The experimental friction peaks had an average width of $6.0^{\circ}$. This corresponds to an estimated diameter of roughly 10 atomic spacings, or approximately $N=100$ atoms. Figure 13(a) shows the experimental friction data ${ }^{19}$ together with the calculations for a symmetric 96-atom flake. Due to the elasticity of the tip in the experiment, the effective spring constant of the force sensor had been lowered from $5.75 \mathrm{~N} / \mathrm{m}$ to $1.80 \mathrm{~N} / \mathrm{m}$ (see Refs. 20 and 21). Slightly better fits to the experiment were obtained for this spring constant in the calculation, if $V_{0}$ was lowered from $0.52 \mathrm{eV}$ to $0.32 \mathrm{eV}$. Most noticeably, modest side peaks have developed next to the main peaks, resulting from incomplete cancellation of forces.

In the experiment, the force patterns were found to rotate as a function of flake orientation. This shows that the flake
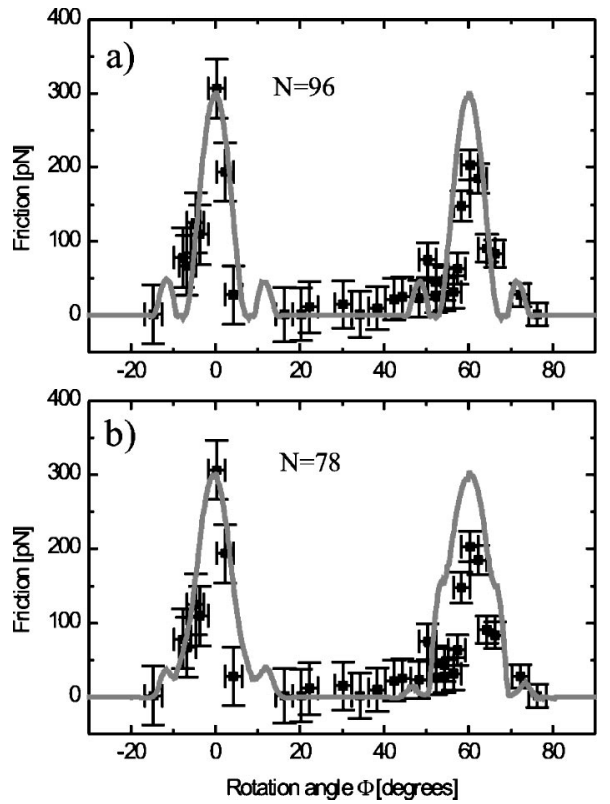

FIG. 13. The data points in both panels show the average friction force versus the rotation angle measured in Ref. 21. The curve through the data points shows the calculated friction force from the Tomlinson model for (a) a symmetric 96-atom flake and (b) an asymmetric 78-atom flake. The calculations were performed for a pulling direction $\Theta=70^{\circ}$ (see text).

was not symmetric. The asymmetry was used to estimate the experimental pulling direction at $\Theta=70^{\circ}$. Comparing the calculated friction versus flake orientation curves with the experimental friction data, we conclude that the 30- and 56atom flakes were too asymmetric. For the 78-atom flake the fit to the experiment is slightly worse than for the symmetric flake, as is illustrated in Fig. 13(b). We conclude that the flake is only mildly asymmetric, in between the 96- and 78atom shapes.

Different peak heights at $0^{\circ}$ and $60^{\circ}$ orientation angle, as found in the experiment, cannot be expected in our calculation, since the simple potential that is used to model the graphite surface and the flake, has $60^{\circ}$ rotational symmetry. This potential only models the interaction between a singlelayer flake and the first layer of the substrate. It ignores the more subtle, long-range interactions that result from the staggered lower graphite layers, most importantly the second layer. As a consequence, a real graphite surface contains two different types of sites for carbon atoms: $A$-type atoms have a direct neighbor in the second layer, and $B$-type atoms do not. This changes the $60^{\circ}$ rotational symmetry of the substrate into $120^{\circ}$ symmetry. If the flake consists of a single graphite layer, the averaging over forward and backward scan lines should restore $60^{\circ}$ symmetry in the friction measurements. However, if the flake consists of two or more graphite layers, the friction signal should only have threefold rotational symmetry. The deviation from sixfold symmetry should, however, be relatively weak, since it originates only from interactions over a distance of three graphite layers.

Since $k_{b} T$ at room temperature is on the order of several percent of the total interaction energies used in the calculations presented here, thermal activation can have a noticeable 
effect on the friction. For example, thermally activated jumps can occur, resulting in earlier tip jumps, and introducing a velocity dependence. A Tomlinson model with a thermal energy term has been used by Gnecco and co-authors ${ }^{27}$ to explain an experimentally observed velocity dependence of the friction.

He et al. have shown in an MD simulation that third bodies, such as hydrocarbon molecules, can cause locking of two surfaces that deform elastically. ${ }^{28}$ This results in static friction, that depends only slightly on the orientational alignment of the two surfaces. By contrast, in our experiments, the friction displayed a dramatic dependence on the relative orientation of the two lattices. This suggests that third bodies did not play a major role in the experiments. ${ }^{19}$

Finally, we discuss the rigidity of the flake and the substrate. Graphite consists of stacked sheets of carbon atoms, separated by a relatively large distance. The van der Waals forces between sheets are weak when compared to the covalent bonding between atoms within the sheet. In other words, the bonding within a single layer is strong when compared to the interaction between layers. This causes the high Young's modulus in the direction parallel to the sheets. Calculations have been performed on double-walled carbon nanotubes (CNT's), where the outer layer incommensurably slides over the inner layer, for both rigid and relaxed layers. ${ }^{29}$ Within the range of sizes studied, relaxation only induced moderate changes. This was attributed to the extreme rigidity of the graphite layers and the weakness of the interlayer interaction. Recently, experimentally observed rolling, rotating, and sliding of CNT's on a graphite surface, ${ }^{30}$ have been modelled successfully, ${ }^{31}$ assuming the CNT's to be rigid. The force needed to rotate a CNT when out of registry with the substrate was very small. Sharp, unique energy minima were found for different types of CNT's as a function of the orientation of the tube axis with respect to the surface lattice.

In spite of the high rigidity of graphite layers, when the size of a graphite flake exceeds a critical value, breakdown of superlubricity can be expected to occur. The in-plane elasticity will eventually be large enough for the flake to distort to improve the registry within finite domains separated by some type of domain walls. Motion of the flake will then be equivalent to the displacement of these walls, which will introduce a new channel for energy dissipation, and thereby remove the superlubricity.

\section{SUMMARY}

In summary, we have set up a Tomlinson model, describing a rigid $\mathrm{N}$-atom cluster with the symmetry of a graphite flake that was moved through a two-dimensional sinusoidal potential representing the graphite surface. The calculated friction force shows high friction and near-zero friction, depending on the (in)commensurability between the two lattices. By changing $N$, we vary the width of the peak in the friction vs orientation plot, which has allowed us to fit the measurements. The calculations revealed that the shapes of the high-friction peaks depend on the precise shape of the flake, and suggest that the flake in the experiments ${ }^{19-21}$ has been slightly asymmetric.

\section{ACKNOWLEDGMENTS}

The authors wish to thank S. Yu. Krylov for stimulating discussions. This work is part of the research program of the Stichting voor Fundamenteel Onderzoek der Materie and was made possible by financial support of the Nederlandse Organisatie voor Wetenschappelijk Onderzoek (NWO).
*Present address: IAVF Antriebstechnik AG, Im Schlehert 32, 76187 Karlsruhe, Germany.

†Electronic address: frenken@phys.leidenuniv.nl

${ }^{1}$ C. M. Mate, G. M. McClelland, R. Erlandsson, and S. Chiang, Phys. Rev. Lett. 59, 1942 (1987).

${ }^{2}$ M. Dienwiebel, J. A. Heimberg, T. Zijlstra, E. van der Drift, D. J. Spaanderman, E. de Kuyper, L. Crama, D. G. van Loon, and J. W. M. Frenken, Rev. Sci. Instrum. (to be published).

${ }^{3}$ G. M. McClelland and S. R. Cohen, in Chemistry and Physics of Solid Surfaces VII, edited by R. Vanselow and R. Rowe (Springer-Verlag, Berlin, 2001), p. 419.

${ }^{4}$ G. A. Tomlinson, Philos. Mag. 7, 905 (1929).

${ }^{5}$ L. Consoli, H. J. F. Knops, and A. Fasolino, Phys. Rev. Lett. 85, 302 (2000).

${ }^{6}$ Y. I. Frenkel and T. Kontorova, Zh. Eksp. Teor. Fiz. 8, 1340 (1938).

${ }^{7}$ M. Weiss and F.-J. Elmer, Phys. Rev. B 53, 7539 (1996).

${ }^{8}$ M. O. Robbins and M. H. Müser, in Modern Tribology Handbook, edited by B. Bhushan (CRC Press, Boca Raton, FL, 2001), pp. 717-765; cond-mat/0001056 (unpublished).

${ }^{9}$ J. B. Sokoloff, Phys. Rev. B 42, 760 (1990).

${ }^{10}$ D. Tománek, W. Zhong, and H. Thomas, Europhys. Lett. 15, 887
(1991).

${ }^{11}$ M. Hirano and K. Shinjo, Phys. Rev. B 41, 11837 (1990).

${ }^{12}$ K. Shinjo and M. Hirano, Surf. Sci. 283, 473 (1993).

${ }^{13}$ H. Hölscher, U. D. Schwarz, O. Zwörner, and R. Wiesendanger, Phys. Rev. B 57, 2477 (1998).

${ }^{14}$ J. W. J. Kerssemakers, Ph.D. thesis, University of Groningen, The Netherlands, 1997.

${ }^{15}$ N. Sasaki, K. Kobayashi, and M. Tsukada, Phys. Rev. B 54, 2138 (1996).

${ }^{16}$ N. Sasaki, M. Tsukada, S. Fujisawa, Y. Sugawara, S. Morita, and K. Kobayashi, Phys. Rev. B 57, 3785 (1998).

${ }^{17}$ T. Gyalog and H. Thomas, Europhys. Lett. 37, 195 (1997).

${ }^{18}$ S. Morita, S. Fujisawa, and Y. Sugawara, Surf. Sci. Rep. 23, 1 (1996).

${ }^{19}$ M. Dienwiebel, G. S. Verhoeven, N. Pradeep, J. W. M. Frenken, J. A. Heimberg, and H. W. Zandbergen, Phys. Rev. Lett. 92, 126101 (2004).

${ }^{20}$ M. Dienwiebel, Ph.D. thesis, Leiden University, The Netherlands, 2003.

${ }^{21}$ M. Dienwiebel, N. Pradeep, G. S. Verhoeven, J. W. M. Frenken, and H. W. Zandbergen (in preparation).

${ }^{22}$ M. R. Sørensen, K. W. Jacobsen, and P. Stolze, Phys. Rev. B 53, 
2101 (1996).

${ }^{23}$ P. E. Sheehan and C. M. Lieber, Science 272, 1158 (1996).

${ }^{24}$ K. Miura and S. Kamiya, Europhys. Lett. 58, 610 (2002).

${ }^{25}$ W. A. Steele, Surf. Sci. 36, 317 (1973).

${ }^{26} \mathrm{~S}$. Y. Krylov (private communication).

${ }^{27}$ E. Gnecco, R. Bennewitz, T. Gyalog, C. Loppacher, M. Bammerlin, E. Meyer, and H.-J. Güntherodt, Phys. Rev. Lett. 84, 1172 (2000).
${ }^{28}$ G. He, M. H. Müser, and M. O. Robbins, Science 284, 1650 (1999).

${ }^{29}$ A. N. Kolmogorov and V. H. Crespi, Phys. Rev. Lett. 85, 4727 (2000).

${ }^{30}$ M. R. Falvo, R. M. Taylor II, A. Helser, V. Chi, F. P. Brooks, Jr., S. Washburn, and R. Superfine, Nature (London) 397, 236 (1999).

${ }^{31}$ A. Buldum and J. P. Lu, Phys. Rev. Lett. 83, 5050 (1999). 